Provided for non-commercial research and educational use only. Not for reproduction or distribution or commercial use.

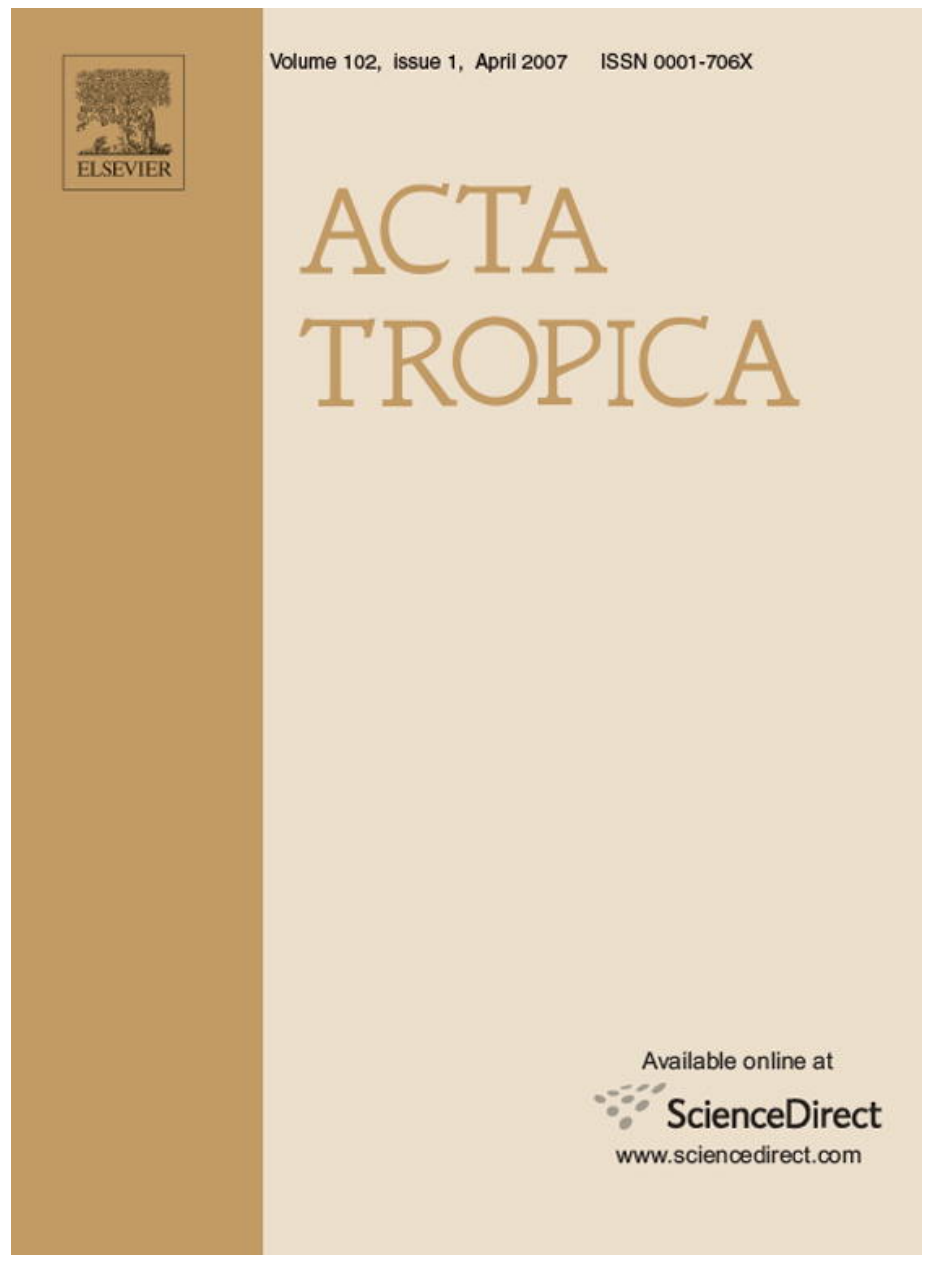

This article was originally published in a journal published by Elsevier, and the attached copy is provided by Elsevier for the author's benefit and for the benefit of the author's institution, for non-commercial research and educational use including without limitation use in instruction at your institution, sending it to specific colleagues that you know, and providing a copy to your institution's administrator.

All other uses, reproduction and distribution, including without limitation commercial reprints, selling or licensing copies or access,

or posting on open internet sites, your personal or institution's website or repository, are prohibited. For exceptions, permission may be sought for such use through Elsevier's permissions site at: 
Short communication

\title{
Label-free detection of Babesia bovis infected red blood cells using impedance spectroscopy on a microfabricated flow cytometer
}

\author{
Claudia Küttel $^{\mathrm{a}, *}$, Elisabete Nascimento ${ }^{\mathrm{b}}$, Nicolas Demierre ${ }^{\mathrm{a}}$, Tiago Silva ${ }^{\mathrm{b}}$, \\ Thomas Braschler $^{\mathrm{a}}$, Philippe Renaud ${ }^{\mathrm{a}}$, Abel G. Oliva ${ }^{\mathrm{b}}$ \\ ${ }^{a}$ Laboratory of Microsystems, Ecole Polytechnique Fédérale de Lausanne, 1015 Lausanne, Switzerland \\ b Instituto de Biologia Experimental e Tecnológica/ITQB, Universidade Nova de Lisboa, 2781-901 Oeiras, Portugal \\ Received 20 November 2006; received in revised form 7 March 2007; accepted 8 March 2007 \\ Available online 12 March 2007
}

\begin{abstract}
Impedance spectroscopy is a powerful tool for label-free analysis and characterisation of living cells. In this work, we achieved the detection of Babesia bovis infected red blood cells using impedance spectroscopy on a microfabricated flow cytometer. The cellular modifications caused by the intracellular parasite result in a shift in impedance which can be measured dielectrically. Thus, a rapid cell-by-cell detection with microliter amounts of reagents is possible. Unlike other diagnostic tests, this method does not depend on extensive sample pre-treatment or expensive chemicals and equipment.
\end{abstract}

(C) 2007 Elsevier B.V. All rights reserved.

Keywords: Label-free; Babesia bovis; Impedance spectroscopy; Dielectrophoretic parasite detection; Infected erythrocytes; Microfabricated flow cytometer

\section{Introduction}

Babesia bovis is an intraerythrocytic protozoan parasite responsible for great economic losses in cattle industry. Members of the genus Babesia are transmitted by ixodid ticks to a broad variety of vertebrate hosts, including domestic and wild animals and some species infect humans (Gaffar et al., 2003). B. bovis parasites are endemic in many tropical and subtropical regions worldwide causing anaemia due to extensive haemolysis and other symptoms (Brown et al., 2006).

To identify early infections several methods are known and used for the detection of B. bovis parasites

\footnotetext{
* Corresponding author. Present address: Nanotechnology Group, ETH Zurich, 8092 Zurich, Switzerland. Tel.: +41 44632 8994; fax: +41 446321278 .

E-mail address: kuettel@nano.mavt.ethz.ch (C. Küttel).
}

in whole blood. Among them, the microscopic examination of Giemsa or Acridine Orange stained blood smears is still considered the method of choice since it is rapid and inexpensive (Böse et al., 1995); nevertheless, it requires skilled personnel and difficulties appear in identifying low infection levels. Flow cytometry is able to detect earlier infections but also requires a specific fluorescent dye, e.g. hydroethidine, to stain live parasites present in intact erythrocytes (Wyatt et al., 1991). Other diagnostic methods like, e.g. the immunofluorescence antibody test (IFAT; Homer et al., 2000), enzyme-linked immunosorbent assays (ELISA; Böse et al., 1995), polymerase chain reaction (PCR; Costa-Júnior et al., 2006) or reverse line blotting (RLB; Schnittger et al., 2004) have been developed to increase sensitivity and specificity. However, most of these methods have the drawback of requiring time-consuming sample preparation, expensive reagents or equipment, and staining of live cells 
can cause cell damage (Seidl et al., 1999). A method that overcomes some of these limitations is impedance spectroscopy, also referred to as dielectric spectroscopy. This non-invasive technique can be used for characterisation and analysis of living biological cells and also for quantifying cell concentrations.

Impedance measurements over a wide range of frequencies provide information about cell size, membrane capacitance and cytoplasm properties. A biological cell experiencing an ac electric field is polarised due to accumulation of charges at phase boundaries, for instance, at the interface of the membrane and the suspending liquid (Schwan, 1957; Asami, 2002). The impedance depends on the amount of polarisation and on the frequency and is dissimilar for different kinds of cells. At low frequencies, the cell appears to be non-conducting since the membrane, with its capacitance, forms a considerable barrier to the current. Thus the measured impedance gives information about the cell size since the current mainly flows around the particle. The higher the frequency, the lower the membrane polarisation and more current flows through the cytoplasm revealing the impedance of the cell interior. This technique has already been used for analysis of various cell types, such as blood cells (Asami et al., 1996; Gimsa et al., 1996) and bacteria on a microfluidic chip (Gómez-Sjöberg et al., 2005) as well as on-chip blood cell differentiation (Cheung et al., 2005). Additionally, the protozoan parasite Plasmodium falciparum has been shown to significantly alter the dielectric properties of human red blood cells, and these properties were used for enrichment by free-flow-fractionation (Gascoyne et al., 2004). As B. bovis and P. falciparum life cycles are very similar (Allred and Al-Khedery, 2004; Cooke et al., 2005) changes in the dielectric properties of $B$. bovis infected cells should be comparable to those in P. falciparum infected cells.

In this work we present the results of B. bovis infected cell detection by impedance spectroscopy using a novel microfabricated device, which was characterised in a previous work (Demierre et al., 2007). The rapid cellby-cell analysis does not depend on expensive reagents for sample preparation and does not imply any cell modification by staining. The device is easy to fabricate at low costs and aims to perform conventional macroscopic laboratory methods - cell detection and separation - with high resolution and specificity in reduced timescales and with only microliter amounts of biological sample.

\section{Materials and methods}

For the characterisation of infected cell detection by impedance spectroscopy B. bovis clonal lines M07 and C61411 were used. Infected erythrocytes were cultured in microaerophilus phase (MASP), according to the Levy and Ristic method (Levy and Ristic, 1980). Parasites were cultured in 24-well suspension plates, where a $1.2 \mathrm{ml}$ settled layer of bovine erythrocytes with a packed cell volume (PCV) of $10 \%(\mathrm{v} / \mathrm{v})$ was grown in M-199 culture medium (Gibco), supplemented with $50 \mu \mathrm{g} / \mathrm{ml}$ gentamycin (Gibco), $1 \%$ (v/v) fungizone (Gibco), $20 \mathrm{mM}$ TES (Sigma-Aldrich) and 40\% (v/v) adult bovine serum (kindly provided by Dr. Neto from EZN). Plates were maintained at $37^{\circ} \mathrm{C}$ under a humidified atmosphere of $5 \% \mathrm{CO}_{2}$ in air. Culture media was exchanged daily and the cultures were maintained at a parasitaemia between $1 \%$ and $3 \%$ by splitting and dilution with fresh normal bovine erythrocytes and medium. Parasitaemia was monitored by microscopic examination of Giemsa-stained smears ( $80 \times$ magnification).

For the measurements cells with 1-20\% parasitaemia were suspended at a concentration of $1 \times 10^{5}$ cells $/ \mathrm{ml}$, in a low conductivity buffer solution ( $\mathrm{pH} 7.4$; conductivity of $56 \mathrm{mS} / \mathrm{m}$ ). The low conductivity isotonic buffer was prepared from phosphate buffered saline (PBS; $\mathrm{pH} 7.4$, conductivity $1.2 \mathrm{~S} / \mathrm{m}, 0.22 \mu \mathrm{m}$ filtered; Sigma-Aldrich) by addition of sucrose and deionised water for achievement of the proper osmolarity and conductivity. For negative controls, samples of uninfected bovine erythrocytes and ghost cells prepared from the uninfected erythrocytes were used. Ghosts were prepared by washing normal red blood cells in PBS and suspending them in a hypotonic buffer (PBS; pH 8.0, $0.22 \mu \mathrm{m}$ filtered), following incubation at $4{ }^{\circ} \mathrm{C}$ for $20-30 \mathrm{~min}$ to burst the cells. The cells were centrifuged at $850 \times g$ for $10 \mathrm{~min}$ and then washed twice in hypotonic buffer. The membranes were suspended in PBS and incubated at room temperature for $1 \mathrm{~h}$ to reseal the membrane sacks.

B. bovis infected erythrocyte cultures were prepared for fluorescence microscopy by incubation for $20 \mathrm{~min}$ with $25 \mu \mathrm{g} / 10^{5}$ cells $/ \mathrm{ml}$ of $3,3^{\prime}$-dihexyloxacarbocyanine iodide $\left(\mathrm{DiOC}_{6}\right.$; Molecular Probes), at room temperature, in the dark. This reagent is a potentiometric probe (permeable membrane cation) that accumulates in parasites, as a result of the transmembrane potential (Gascoyne et al., 2002). Parasites viability was measured before and after impedance measurements with $20 \mu \mathrm{g} / 10^{5}$ cells $/ \mathrm{ml}$ 6-carboxyfluorescein diacetate (CFDA; Sigma-Aldrich), incubated during $30 \mathrm{~min}$ at $20^{\circ} \mathrm{C}$ in the dark. The CFDA fluorophore, which is nonfluorescent, permeates membranes and only then hydrolysis of the ester bonds results in formation of a highly fluorescent green fluorophore which is trapped within cellular structures possessing intact membranes. The cell suspension was resuspended in PBS after cen- 
trifugation at $850 \times g$ for $10 \mathrm{~min}$ and examined using fluorescence microscopy (Franssen et al., 2003).

The microfluidic device presented first by Demierre et al. (2007) consists of two planar electrode pairs to differentially measure the cells passing through a microfluidic channel. The cells were driven through the microfluidic channel by an applied pressure gradient. An ac electric potential at a defined set of frequencies is applied to the two pairs of electrodes. This potential applied over each pair of electrodes results in an electric field and every passing cell crossing this electric field modifies temporarily the impedance as well as the current which allows detecting membrane and cytoplasm properties. The electronic circuit converts the currents in potentials and compares them in order to perform a differential measurement of the impedance between the two electrode pairs to avoid signal drift. A more detailed description of the measurement system was given by Gawad et al. (2001).

\section{Results}

Results obtained from B. bovis infected red blood cell cultures measured by impedance spectroscopy were displayed in a scatterplot of imaginary part versus real part of the signal amplitude, both at the frequency of $8.7 \mathrm{MHz}$ (Fig. 1). The results show that changes of the signal amplitudes at a frequency of $8.7 \mathrm{MHz}$ are clearly observable for samples containing infected cells (Fig. 1a). These changes at high frequency indicate a variation of the dielectric properties of the cytoplasm. Impedance measurements at lower frequencies in the range of $10 \mathrm{kHz}$ to $1 \mathrm{MHz}$, which have been shown to be related to information about cell size and membrane properties, did not produce such obvious changes (figure not shown). Samples of uninfected bovine erythrocytes (Fig. 1b) and erythrocyte ghosts synthesized from uninfected bovine erythrocytes (Fig. 1c) were used as negative controls.

The scatterplots presented are divided in three areas, uninfected RBCs (uRBC), parasitised RBCs (pRBC) and ghost RBCs (gRBC). The latter are red blood cells which burst as a consequence of cell ageing, parasitic rupture or medium properties; the cytoplasm containing haemoglobin was released and the membrane sack resealed with medium inside it. The majority of the uninfected control sample (Fig. 1b) is plotted in the corresponding area 'uRBC'. This control sample has a negligible amount of signals in the 'pRBC' region due to signal noise and data points in the ' $\mathrm{gRBC}$ ' region are likely due to ghost cells, which in this case, appeared as a consequence of cell ageing. B. bovis infected samples containing parasitised cells, as well as uninfected cells (Fig. 1a), present a high percentage of signals in the 'pRBC' region, showing a general signal shift to different phase angles indicative of the degree of infection or the amount of parasites per host cell. The amount of ghosts - formed by parasitic rupture - in the ' $\mathrm{gRBC}$ ' domain increased considerably when compared to the negative control of uninfected cells. Furthermore, the population of the control of ghost cells (Fig. 1c) is clearly displayed in the ' $\mathrm{gRBCs}$ ' region. A slight difference, however, does exist between signals obtained from ghosts in the infected sample and ghosts in the control

(a) Real part of signal amplitude (V)

(b) Real part of signal amplitude ( $\mathrm{V}$ )

(c) Real part of signal amplitude (V)
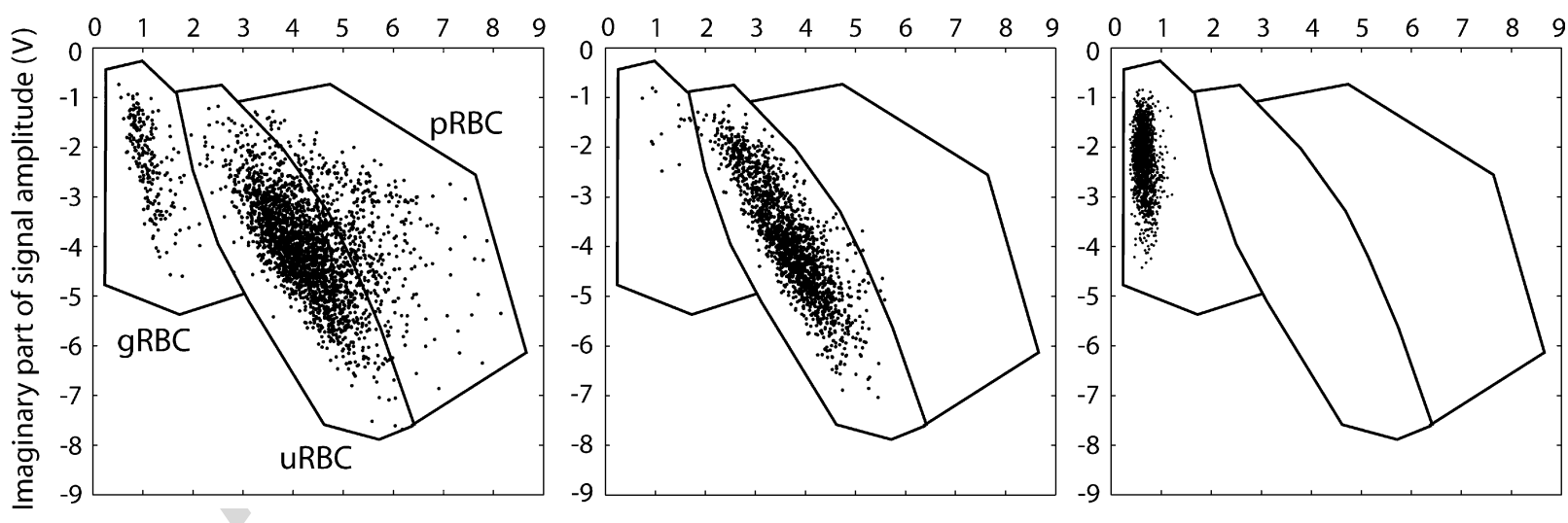

Fig. 1. Representative scatterplots of impedance spectroscopy measurements display the dielectric changes in Babesia bovis infected cells. Scatterplots of (a) a B. bovis infected sample of bovine erythrocytes, (b) a control of uninfected bovine erythrocytes and (c) a control of uninfected erythrocyte ghosts. The areas for uninfected RBCs ( $\mathrm{uRBC}$ ), parasitised RBCs (pRBC) and ghost RBCs ( $\mathrm{gRBC}$ ) are marked in the three scattergrams. The signal amplitudes are split into real $(X)$ and imaginary $(Y)$ parts, which are measured in volts. Signal differences are visible in the signal phase which is defined as $\arctan (Y / X)$. All the measurements were performed with an ac electric field of frequency $8.7 \mathrm{MHz}$ to detect changes in dielectric properties of the cells due to the intracellular parasite. The number of cells used was 2700 in (a), 1700 in (b) and 1500 in (c) after extraction of invalid signals from the samples. 
sample. When the ghost cell still contains an intracellular parasite, the total impedance of the cell interior is slightly bigger than that of an empty ghost cell. By assigning regions ' $\mathrm{uRBC}$ ' and ' $\mathrm{gRBC}$ ' to uninfected erythrocytes and ghosts, respectively, the ' $\mathrm{pRBC}$ ' region presents signals from parasitised cells. This conclusion was verified by additional measurements with a simultaneous optical assay using fluorescence microscopy of the B. bovis infected samples. Signals of each cell type were identical for fluorescently stained and unstained samples.

The shift in phase for parasitised cells can also be seen in a histogram for cell count when the signal phase at $8.7 \mathrm{MHz}$ is increased from $-90^{\circ}$ to $0^{\circ}$ (Fig. 2). Two additional peaks that reflect sub-populations are distinguishable when comparing the histogram for an infected sample with an uninfected sample. They consist in parasitised RBCs for higher signal phases (between $-30^{\circ}$ and $0^{\circ}$ ) and ghosts for lower signal phase (between $-90^{\circ}$ and $-50^{\circ}$ ) which are clearly not visible in the negative control of healthy cells. As these measurements are performed with ac electric fields, the viability of the parasites could be affected. Nevertheless, viability tests using CFDA fluorescent stains carried out before and after the measurements indicated no visible changes in viability.

\section{Discussion}

The obtained results show that the detection of $B$. bovis infected bovine erythrocytes by impedance spectroscopy is possible when exploiting the changes in the dielectric properties produced by the parasites. Although cells are dielectrically measured, this study does not include the analysis of the cell dielectric properties such as internal and membrane permittivities and conductivities, as it was made by other groups for, e.g. blood cells (Asami et al., 1996) or cellular response to toxicants (Ratanachoo et al., 2002). Most of the surveys that discuss the cell's dielectric properties include several measurement frequencies and work over a longer time-range with a single cell or a cell population using a dielectric model to identify the cell parameters. After preliminary measurements over a sweep of frequencies, our measurements are limited to one or two simultaneously applied frequencies for ideal discrimination between populations. This happens at the cost of modelling the cell characteristics, since models for dielectric cell properties include continuous measurements over a broader frequency range, but it reduces electronic overhead and time. Gascoyne et al. (2004) presented a method for malaria specimen's preparation by dielec-

\section{B. bovis infected RBCs}

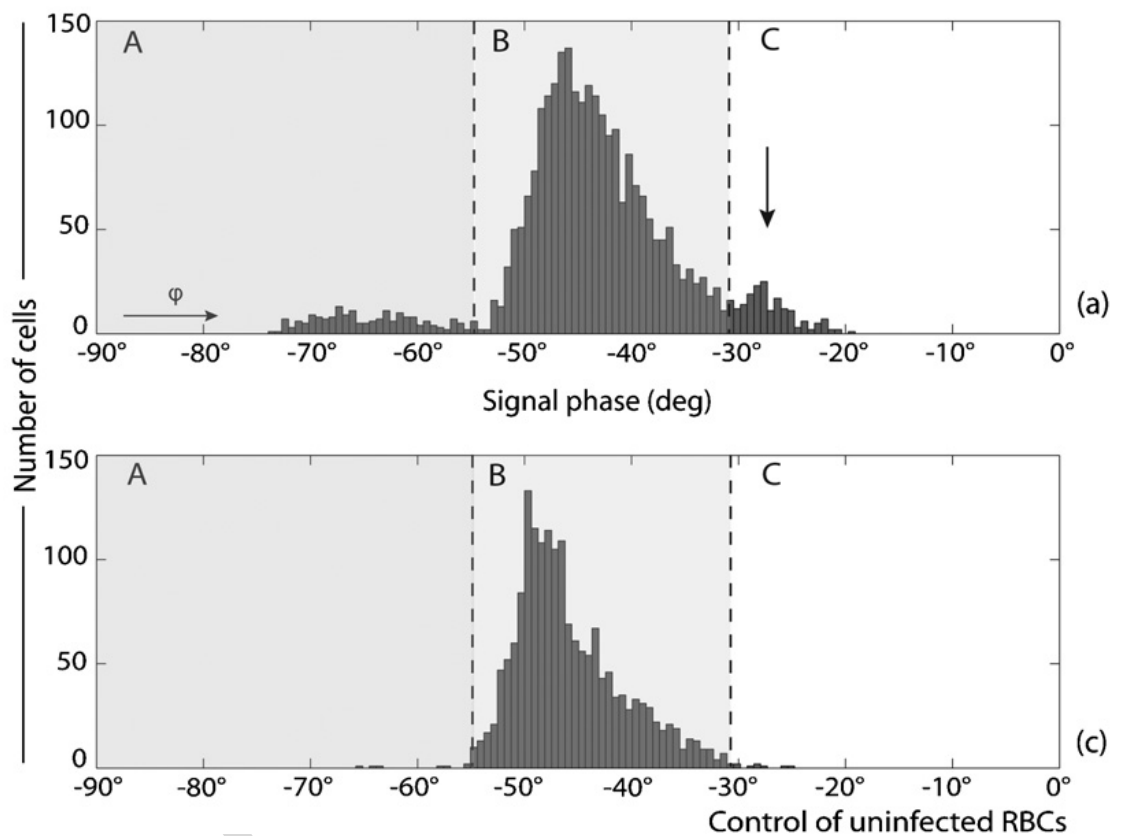

Real part of signal amplitude (V)

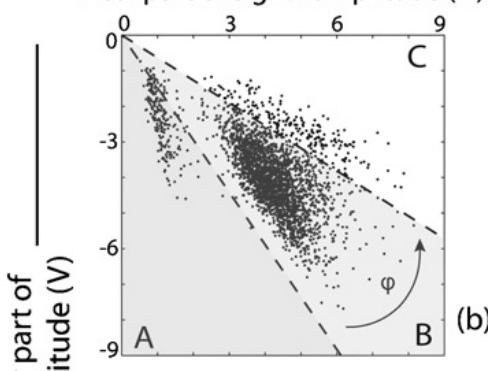

(b)

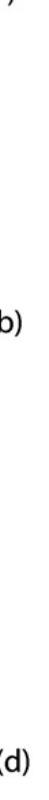

Fig. 2. Analysis of histograms yields detection of parasitised cell sub-population. Histogram of cell counts of signal phases (a) and scattergram (b) displayed for B. bovis infected cells. The same graphs for a negative control of healthy bovine erythrocytes (c, d) are displayed beneath. Regions A-C mark the locations of identified sub-populations of ghost RBCs, uninfected RBCs and parasitised RBCs, respectively. The peak for the parasitised RBC sub-population which is indicated by the arrow in the histogram (a) appears between $-30^{\circ}$ and $0^{\circ}$ while no peak in that region appears in the histogram of the negative control (c). The population peak of the uninfected RBCs in both histograms is located at a signal phase between $-55^{\circ}$ and $-30^{\circ}$. At phases lower than about $-55^{\circ}$, a third peak due to ghost cells can be identified in histogram (a). The angle direction is indicated in (a) and (b). 
trophoretic field-flow-fractionation, demonstrating that faster sample treatment for direct cell separation is possible. This separation method can then be used for subsequent detection by fluorescence based tests or PCR. Although similar principles were employed in this study, the method presented here focused rather on the direct dielectric detection of parasitised cells without the use of any markers. Thus the dielectric cell characteristics were used for fast cell-by-cell analysis rather than for extensive single cell characterisation or enrichment. By adding a stage for sorting, however, the method described can also be used for active cell separation or enrichment for further clinical or biochemical analysis of the parasite in situ. The use of sucrose, Ficoll or Percoll linear gradients and differential centrifugation have been used in the enrichment of B. bovis infected red blood cells, but none of these techniques can be used for in situ analyses.

Bovine babesiosis is a disease that poses particular problems in regions lacking good infrastructure and for this reason could greatly benefit from the development of such a rapid, automated diagnostic method, since the 'gold standard' - detection of stained smears by optical microscopy - usually used for detection of parasites in situ requires experienced personnel and microscopes which are hard to transport to very remote areas. By employing impedance spectroscopy flow cytometry, whole blood samples could even be added without preparation since the dielectric properties of leukocytes or platelets are again different from parasitised cells at certain frequencies. With this the overall measurement time could be reduced and rapid analysis of the blood samples without extensive sample pre-treatment and expensive chemicals or equipment could be provided. So far, our measurements of infected in vitro cultures showed that detection of samples with 1-20\% parasitaemia is possible. Future work with samples from infected animals can show the sensitivity limitations of such a sensor. Additionally, investigations of mixed infections including $B$. bovis and B. bigemina will show the detection limits of the system to discriminate between the two species, since mixed infections often occur in animals from regions where the disease is endemic.

\section{Acknowledgements}

B. bovis clonal lines M07 and C61411 were kindly provided by Dr. Erik de Vries, from the Department of Infectious Diseases and Immunology, Utrecht University, in the Netherlands.

Bovine blood from healthy donors was kindly provided by Dr. Maria Irene Vasques and Dr. João Maria
Neto from Estação Zootécnica Nacional (EZN), in Portugal.

This work was supported by the European Community-funded CellPROM project under the 6th Framework Programme, contract No. NMP4-CT-2004500039 .

\section{References}

Allred, D.R., Al-Khedery, B., 2004. Antigenic variation and cytoadhesion in Babesia bovis and Plasmodium falciparum: different logics achieve the same goal. Mol. Biochem. Parasitol. 134, 2735.

Asami, K., Yonezawa, T., Wakamatsu, H., Koyanagi, N., 1996. Dielectric spectroscopy of biological cells. Bioelectrochem. Bioenerg. 40, $141-145$.

Asami, K., 2002. Characterization of heterogeneous systems by dielectric spectroscopy. Prog. Polym. Sci. 27, 1617-1659.

Böse, R., Jorgensen, W.K., Dalgliesh, R.J., Friedhoff, K.T., de Vos, A.J., 1995. Current state and future trends in the diagnosis of babesiosis. Vet. Paras. 57, 61-74.

Brown, W.C., Norimine, J., Goff, W.L., Suarez, C.E., Mcelwain, T.F., 2006. Prospects for recombinant vaccines against Babesia bovis and related parasites. Paras. Immunol. 28, 315-327.

Cheung, K., Gawad, S., Renaud, P., 2005. Impedance spectroscopy flow cytometry: on-chip label-free cell differentiation. Cytometry A $65,124-132$.

Cooke, B.M., Mohandas, N., Cowman, A.F., 2005. Cellular adhesive phenomena in apicomplexan parasites of red blood cells. Vet. Parasitol. 132, 273-295.

Costa-Júnior, L.M., Rabelo, E.M.L., Martins, O.A., Ribeiro, M.F.B., 2006. Comparison of different direct diagnostic methods to identify Babesia bovis and Babesia bigemina in animals vaccinated with live attenuated parasites. Vet. Parasitol. 139, 231-236.

Demierre, N., Braschler, T., Linderholm, P., Seger, U., Renaud, P., 2007. Characterization and optimization of liquid electrodes for lateral dielectrophoresis. Lab Chip 7, 355-365.

Franssen, F.F.J., Gaffar, F.R., Yatsuda, A.P., de Vries, E., 2003. Characterisation of erythrocyte invasion by Babesia bovis merozoites efficiently released from their host cell after high-voltage pulsing. Microbes Infect. 5, 365-372.

Gaffar, F.R., Franssen, F.F.J., de Vries, E., 2003. Babesia bovis merozoites invade human, ovine, equine, porcine and caprine erythrocytes by sialic acid-dependent mechanism followed by developmental arrest after a single round of cell fission. Int. J. Parasitol. 33, 1595-1603.

Gascoyne, P., Mahidol, C., Ruchirawat, M., Satayavivad, J., Watcharasit, P., Becker, F.F., 2002. Microsample preparation by dielectrophoresis: isolation of malaria. Lab Chip 2, 70-75.

Gascoyne, P., Satayavivad, J., Ruchirawat, M., 2004. Microfluidic approaches to malaria detection. Acta Trop. 89, 357-369.

Gawad, S., Schild, L., Renaud, P., 2001. Micromachined impedance spectroscopy flow cytometer for cell analysis and particle sizing. Lab Chip 1, 76-82.

Gimsa, J., Müller, T., Schnelle, T., Fuhr, G., 1996. Dielectric spectroscopy of single human erythrocytes at physiological ionic strength: dispersion of the cytoplasm. Biophys. J. 71, 495-506.

Gómez-Sjöberg, R., Morisette, D., Bashir, R., 2005. Impedance microbiology-on-a-chip: microfluidic bioprocessor for rapid detection of bacterial metabolism. J. Microelectromech. Syst. 14, 829-838. 
Homer, M., Aguilar-Delfin, I., Telford III, S.R., Krause, P.J., Persing, D.H., 2000. Babesiosis. Clin. Microbiol. Rev. 13, 451469.

Levy, M.G., Ristic, M., 1980. Babesia bovis: continuous cultivation in a microaerophilus stationary phase culture. Science 207, 1218-1220.

Ratanachoo, K., Gascoyne, P.R.C., Ruchirawat, M., 2002. Detection of cellular responses to toxicants by dielectrophoresis. Biochim. Biophys. Acta 1564, 449-458.

Schnittger, L., Yin, H., Qi, B., Gubbels, M.J., Beyer, D., Niemann, S., Jongean, F., Ahmed, J.S., 2004. Simultaneous detection and differentiation of Theileria and Babesia parasites infecting small ruminants by reverse line blotting. Parasitol. Res. 92, 189-196.

Schwan, H.P., 1957. Electrical properties of tissue and cell suspensions. Adv. Biol. Med. Phys. 5, 147-209.

Seidl, J., Knuechel, R., Kunz-Schughart, L.A., 1999. Evaluation of membrane physiology following fluorescence activated or magnetic cell separation. Cytometry 36, 102-111.

Wyatt, C.R., Goff, W., Davis, W.C., 1991. A flow cytometric method for assessing viability of intraerythrocytic hemoparasites. J. Immunol. Meth. 24, 23-30. 